\title{
Article
}

\section{Yet Another New Variant of Szász-Mirakyan Operator}

\author{
Ana Maria Acu ${ }^{1,+}+\mathbb{D}$ and Gancho Tachev ${ }^{2, *,+}$ \\ 1 Department of Mathematics and Informatics, Lucian Blaga University of Sibiu, Str. Dr. I. Ratiu, No. 5-7, \\ 550012 Sibiu, Romania; anamaria.acu@ulbsibiu.ro \\ 2 Department of Mathematics, University of Architecture Civil Engineering and Geodesy, 1046 Sofia, Bulgaria \\ * Correspondence: gtt_fte@uacg.bg \\ + These authors contributed equally to this work.
}

check for

updates

Citation: Acu, A.M.; Tachev, G. Yet Another New Variant of

Szász-Mirakyan Operator. Symmetry 2021, 13, 2018. https://doi.org/

$10.3390 /$ sym 13112018

Academic Editor: Calogero Vetro

Received: 13 August 2021

Accepted: 8 October 2021

Published: 25 October 2021

Publisher's Note: MDPI stays neutral with regard to jurisdictional claims in published maps and institutional affiliations.

\section{Copyright: (C) 2021 by the authors.} Licensee MDPI, Basel, Switzerland. This article is an open access article distributed under the terms and conditions of the Creative Commons Attribution (CC BY) license (https:/ / creativecommons.org/licenses/by/ $4.0 /)$.

\begin{abstract}
In this paper, we construct a new variant of the classical Szász-Mirakyan operators, $M_{n}$ which fixes the functions 1 and $e^{a x}, x \geq 0, a \in \mathbb{R}$. For these operators, we provide a quantitative Voronovskaya-type result. The uniform weighted convergence of $M_{n}$ and a direct quantitative estimate are obtained. The symmetry of the properties of the classical Szász-Mirakyan operator and of the properties of the new sequence is investigated. Our results improve and extend similar ones on this topic, established in the last decade by many authors.
\end{abstract}

Keywords: Szász-Mirakyan operators; weighted approximation; uniform convergence; exponential functions

\section{Introduction}

In the last decade, a lot of papers devoted to modifications of certain positive linear operators (p.l.o), which fix certain exponential functions, have been published. The papers listed in the references are only a small part of all such research works. The start was given by P. King in his famous paper [1] from 2003, where he modified the Bernstein operator to achieve better approximation on some subintervals of $[0,1]$. Later, this method was extended in the papers of Raşa, Aldaz, Kounchev, Render (see [2-16]) and many others. Apart from preservation of monomials, there is an increasing interest to modify the operators of Bernstein, Szász-Mirakyan, Baskakov, Phillips and their Kantorovich and Durrmeyer variants, such that the new modified operators reproduce certain exponential type-functions (see [17-25]).

There are two methods for such modifications. In most of the papers listed in our references (for example see [2,14-16,26]), the authors modify the basis functions of SzászMirakyan operator

$$
S_{n, k}(x)=e^{-n x} \frac{(n x)^{k}}{k !}, k \geq 0,
$$

where instead of $x$, they take an appropriate positive function $\alpha_{n}(x)$, i.e.,

$$
S_{n, k}\left(\alpha_{n}(x)\right)=e^{-n \alpha_{n}(x)} \frac{\left(n \alpha_{n}(x)\right)^{k}}{k !} .
$$

In [2], even two positive functions $\alpha_{n}(x), \beta_{n}(x)$ are used to have as basis functions

$$
e^{-n \alpha_{n}(x)} \frac{\left(n \beta_{n}(x)\right)^{k}}{k !}, k \geq 0 .
$$

The second method consists of modifying the argument of the function $f(x)$ to be approximated (in the spirit of King's paper). For example Aral, Inoan and Raşa in [11] studied the approximation properties of the operator 


$$
S_{n}^{\rho}(f ; x)=e^{-n \rho(x)} \sum_{k=0}^{\infty}\left(f \circ \rho^{-1}\right)\left(\frac{k}{n}\right) \frac{(n \rho(x))^{k}}{k !},
$$

where $\rho$ satisfies certain conditions.

Our method combines the two ideas mentioned above, i.e., we modify the basis functions $S_{n, k}(x)$ and simultaneously multiply $f(x)$ by certain exponentials.

Before introducing our new operators, we recall some notations from Gonska et al. [26]. In [26], the following modified Szász-Mirakyan operator was studied

$$
R_{n, 1}^{*}(f ; x)=e^{-n \alpha_{n, 1}(x)} \sum_{k=0}^{\infty} \frac{\left(n \alpha_{n, 1}(x)\right)^{k}}{k !} f\left(\frac{k}{n}\right), x \geq 0, n \in \mathbb{N},
$$

where the conditions

$$
R_{n, 1}^{*}(1 ; x)=1, R_{n, 1}^{*}\left(e^{a t} ; x\right)=e^{a x}
$$

are satisfied for

$$
\alpha_{n, 1}(x)=\frac{a x}{n\left(e^{a / n}-1\right)}, a>0 .
$$

The condition $a>0$ was imposed in [26] because it was used further in the paper. On the other hand, we observe that $\alpha_{n, 1}(x)>0$ for all $a \neq 0$, including also $a<0$. Then, this class of operators which preserve the functions 1 and $e^{a x}$ can be extended for $a<0$. Denote by

$$
R_{n, 2}^{*}(f ; x)=e^{-n \alpha_{n, 2}(x)} \sum_{k=0}^{\infty} \frac{\left(n \alpha_{n, 2}(x)\right)^{k}}{k !} f\left(\frac{k}{n}\right), x \geq 0, n \in \mathbb{N},
$$

where

$$
\alpha_{n, 2}(x)=\frac{-a x}{n\left(e^{-a / n}-1\right)}, a>0 .
$$

Note that the operators $R_{n, 2}^{*}$ verify the conditions

$$
R_{n, 2}^{*}(1 ; x)=1, R_{n, 2}^{*}\left(e^{-a t} ; x\right)=e^{-a x} .
$$

Motivated by the results from [26], in this paper, we introduce operators which preserve the functions 1 and $e^{a x}$ for all $a \in \mathbb{R} \backslash\{0\}$. The results from [26] are improved considering approximation properties in weighted spaces. Moreover, some quantitative estimates for approximation by the new operators are obtained.

Combining the techniques described above, we introduce the operators

$$
\begin{aligned}
M_{n}(f ; x) & =e^{a x} R_{n, 2}^{*}\left(f(t) e^{-a t} ; x\right) \\
& =e^{a x} \cdot e^{-n \alpha_{n, 2}(x)} \sum_{k=0}^{\infty} \frac{\left(n \alpha_{n, 2}(x)\right)^{k}}{k !} f\left(\frac{k}{n}\right) e^{-a \frac{k}{n}}, a>0 .
\end{aligned}
$$

We have

$$
M_{n}(1 ; x)=e^{a x} R_{n, 2}^{*}\left(e^{-a t} ; x\right)=1 ; M_{n}\left(e^{a t} ; x\right)=e^{a x} R_{n, 2}^{*}(1 ; x)=e^{a x}
$$

A new class of operators which preserve the functions 1 and $e^{-a x}, a>0$ can be introduced as follows

$$
\begin{aligned}
N_{n}(f ; x) & =e^{-a x} R_{n, 1}^{*}\left(f(t) e^{a t} ; x\right) \\
& =e^{-a x} \cdot e^{-n \alpha_{n, 1}(x)} \sum_{k=0}^{\infty} \frac{\left(n \alpha_{n, 1}(x)\right)^{k}}{k !} f\left(\frac{k}{n}\right) e^{a \frac{k}{n}}, a>0 .
\end{aligned}
$$

It is easy to prove that 


$$
N_{n}(1 ; x)=e^{-a x} R_{n, 1}^{*}\left(e^{a t} ; x\right)=1 ; N_{n}\left(e^{-a t} ; x\right)=e^{-a x} R_{n, 1}^{*}(1 ; x)=e^{-a x} .
$$

In order to describe the rate of convergence, a quantitative Voronovskaya-type result for the operators $M_{n}$ is proved in Section 2. Section 3 is devoted to the approximation order of operators $M_{n}$ applied to unbounded functions with certain exponential growth. Some quantitative estimates for approximation by $M_{n}$ are obtained in Section 4 . Similar results can be obtained immediately for $N_{n}$.

\section{Voronovskaja-Type Estimate}

In this section, we provide a quantitative Voronovskaya-type theorem. Let $C^{*}[0, \infty)$ be the class of real-valued continuous functions $f(x)$, for which $\lim _{x \rightarrow \infty} f(x)$ exists and is finite, equipped with the uniform norm.

In order to give a Voronovskaya-type estimate, we consider the following modulus of continuity:

$$
\omega^{*}(f ; \delta):=\sup _{\substack{x, y \geq 0 \\\left|e^{-x}-e^{-t}\right| \leq \delta}}|f(x)-f(t)| .
$$

Denote $\mu_{n}^{k}(x)=M_{n}\left((t-x)^{k} ; x\right), k=0,1,2, \ldots$

Lemma 1. We have

$$
\begin{aligned}
& \mu_{n}^{0}(x)=1, \\
& \mu_{n}^{1}(x)=\frac{x\left(n e^{\frac{a}{n}}-a-n\right)}{n\left(1-e^{\frac{a}{n}}\right)}, \\
& \mu_{n}^{2}(x)=\frac{\left(n e^{\frac{a}{n}}-n-a\right)^{2}}{n^{2}\left(e^{\frac{a}{n}}-1\right)^{2}}+\frac{a x}{n^{2}\left(e^{\frac{a}{n}}-1\right)} .
\end{aligned}
$$

Moreover,

$$
\begin{aligned}
& \lim _{n \rightarrow \infty} n \mu_{n}^{1}(x)=-\frac{1}{2} a x, \\
& \lim _{n \rightarrow \infty} n \mu_{n}^{2}(x)=x .
\end{aligned}
$$

Theorem 1. Let $f, f^{\prime \prime} \in C^{*}[0, \infty)$. Then

$$
\begin{gathered}
\left|n\left[M_{n}(f ; x)-f(x)\right]+\frac{a}{2} x f^{\prime}(x)-\frac{1}{2} x f^{\prime \prime}(x)\right| \\
\leq\left|A_{n}(x)\right| \cdot\left|f^{\prime}(x)\right|+\left|B_{n}(x)\right| \cdot\left|f^{\prime \prime}(x)\right|+2\left[2 B_{n}(x)+x+r_{n}(x)\right] \omega^{*}\left(f^{\prime \prime}, n^{-1 / 2}\right)
\end{gathered}
$$

holds for any $x \in[0, \infty)$, where

$$
\begin{aligned}
& A_{n}(x)=n \mu_{n}^{1}(x)+\frac{1}{2} a x, \\
& B_{n}(x)=\frac{1}{2}\left(n \mu_{n}^{2}(x)-x\right), \\
& r_{n}(x)=n^{2} \sqrt{M_{n}\left(\left(e^{-x}-e^{-t}\right)^{4} ; x\right)} \sqrt{M_{n}\left((t-x)^{4} ; x\right)} .
\end{aligned}
$$

Proof. Using Taylor expansion of $f$ at the point $x \in[0, \infty)$, we obtain 


$$
\begin{gathered}
\left|n\left[M_{n}(f ; x)-f(x)\right]+\frac{1}{2} a x f^{\prime}(x)-\frac{1}{2} x f^{\prime \prime}(x)\right| \\
\leq\left|A_{n}(x)\right| \cdot\left|f^{\prime}(x)\right|+\left|B_{n}(x)\right| \cdot\left|f^{\prime \prime}(x)\right|+\left|n M_{n}\left(h(t, x)(t-x)^{2} ; x\right)\right|,
\end{gathered}
$$

where $h(t, x):=\frac{f^{\prime \prime}(\xi)-f^{\prime \prime}(x)}{2}$ and $\xi$ is a number between $x$ and $t$.

Applying the technique used in [26], we can write

$$
|h(t, x)| \leq\left(1+\frac{\left(e^{-x}-e^{-t}\right)^{2}}{\delta^{2}}\right) \omega^{*}\left(f^{\prime \prime}, \delta\right), \delta>0 .
$$

Using this and Cauchy-Schwarz inequality and considering $\delta=n^{-1 / 2}$, we obtain

$$
\begin{aligned}
n M_{n}\left(|h(t, x)|(t-x)^{2}, x\right) & \leq 2 n \omega^{*}\left(f^{\prime \prime}, \delta\right) \cdot M_{n}\left((t-x)^{2} ; x\right) \\
& +\frac{2 n}{\delta^{2}} \omega^{*}\left(f^{\prime \prime}, \delta\right) \sqrt{M_{n}\left(\left(e^{-x}-e^{-t}\right)^{4} ; x\right)} \sqrt{M_{n}\left((t-x)^{4} ; x\right)} \\
& =2\left[2 B_{n}(x)+x+r_{n}(x)\right] \omega^{*}\left(f^{\prime \prime}, n^{-1 / 2}\right),
\end{aligned}
$$

and the proof is complete.

Corollary 1. Let $f, f^{\prime \prime} \in C^{*}[0, \infty)$. Then

$$
\lim _{n \rightarrow \infty} n\left[M_{n}(f ; x)-f(x)\right]=-\frac{a}{2} x f^{\prime}(x)+\frac{1}{2} x f^{\prime \prime}(x),
$$

for any $x \in[0, \infty)$.

To simplify the calculations in the next two sections, we will consider the case $a=1$. Similar results can be obtained for all $a \in \mathbb{R} \backslash\{0\}$.

\section{Weighted Uniform Approximation by $M_{n}$}

In this section, we study the approximation of unbounded functions satisfying certain exponential growth. Set $\varphi(x)=1+e^{x}, x \in \mathbb{R}^{+}$, and consider the following weighted spaces

$$
\begin{aligned}
& B_{\varphi}\left(\mathbb{R}^{+}\right)=\left\{f: \mathbb{R}^{+} \rightarrow \mathbb{R}:|f(x)| \leq M_{f} \varphi(x), x \geq 0\right\}, \\
& C_{\varphi}\left(\mathbb{R}^{+}\right)=C\left(\mathbb{R}^{+}\right) \cap B_{\varphi}\left(\mathbb{R}^{+}\right), \\
& C_{\varphi}^{k}\left(\mathbb{R}^{+}\right)=\left\{f \in C_{\varphi}\left(\mathbb{R}^{+}\right): \lim _{x \rightarrow \infty} \frac{f(x)}{\varphi(x)}=k_{f}, \text { exists and is finite }\right\},
\end{aligned}
$$

where $M_{f}, k_{f}$ are constants depending on $f$. All three spaces are normed with the norm

$$
\|f\|_{\varphi}=\sup \frac{|f(x)|}{\varphi(x)} .
$$

Let

$$
\omega_{\varphi}(f, \delta):=\sup _{\substack{x, y \geq 0 \\\left|e^{\frac{x}{2}}-e^{\frac{y}{2}}\right| \leq \delta}} \frac{|f(x)-f(y)|}{\varphi(x)+\varphi(y)},
$$

for each $f \in C_{\varphi}\left(\mathbb{R}^{+}\right)$and for every $\delta \geq 0$. 
Further, from (5), we obtain

$$
\begin{aligned}
M_{n}(f ; x) & =e^{x} R_{n, 2}^{*}\left(\frac{f(t)}{1+e^{t}}\left(1+e^{t}\right) e^{-t} ; x\right) \\
& \leq e^{x}\|f\|_{\varphi} R_{n, 2}^{*}\left(e^{-t}+1 ; x\right) \\
& =e^{x}\|f\|_{\varphi}\left(e^{-x}+1\right)=\|f\|_{\varphi}\left(1+e^{x}\right) .
\end{aligned}
$$

Consequently, we have

$$
\left\|M_{n} f\right\|_{\varphi} \leq\|f\|_{\varphi},
$$

and conclude that $M_{n}$ maps $C_{\varphi}\left(\mathbb{R}^{+}\right)$to $C_{\varphi}\left(\mathbb{R}^{+}\right)$.

Theorem 2. Let $\varphi(x)=1+e^{x}$. For each function $f \in C_{\varphi}^{k}\left(\mathbb{R}^{*}\right)$, we have

$$
\lim _{n \rightarrow \infty}\left\|M_{n} f-f\right\|_{\varphi}=0 .
$$

Proof. Following the general result obtained by Gadziev [27], to conclude that for each function $f \in C_{\varphi}^{k}\left(\mathbb{R}^{+}\right)$we have

$$
\lim _{n \rightarrow \infty}\left\|M_{n} f-f\right\|_{\varphi}=0
$$

it is enough to verify the three conditions

$$
\lim _{n \rightarrow \infty}\left\|M_{n}\left(e^{i t} ; x\right)-e^{i x}\right\|_{\varphi}=0, i=0, \frac{1}{2}, 1 .
$$

Now, following (6) and according to the Korovkin-type theorem established in [27], we need to verify

$$
\lim _{n \rightarrow \infty}\left\|M_{n}\left(e^{\frac{t}{2}} ; x\right)-e^{\frac{x}{2}}\right\|_{\varphi}=0 .
$$

Consequently, from (5) we may write

$$
\begin{aligned}
M_{n}\left(e^{\frac{t}{2}} ; x\right) & =e^{x} e^{-n \alpha_{n, 2}(x)} \cdot e^{n \alpha_{n, 2}(x) e^{-\frac{1}{2 n}}} \\
& =e^{x} \cdot e^{n \alpha_{n, 2}(x)\left(-1+e^{-\frac{1}{2 n}}\right)}=e^{x} \cdot e^{-\frac{x}{1+e^{-\frac{1}{2 n}}}} \\
& =e^{x\left(\frac{1}{1+e^{1 / 2 n}}\right)} .
\end{aligned}
$$

If we set $g(x)=\frac{\left|M_{n}\left(e^{\frac{t}{2}} ; x\right)-e^{\frac{x}{2}}\right|}{1+e^{x}}$ we obtain



Obviously,

$$
\sup _{x \in[0, \infty)} \frac{e^{x}}{1+e^{x}}=1 .
$$

Further setting $y=e^{-\frac{x}{2}} \in(0,1]$, we need to estimate $\sup _{y \in(0,1]} p(y)$, where

$$
p(y)=y-y^{\frac{2 e^{1 / 2 n}}{1+e^{1 / 2 n}}}
$$


We have $p(0)=p(1)=0$ and $p^{\prime}(y)=1-\frac{2 e^{\frac{1}{2 n}}}{1+e^{\frac{1}{2 n}}} y^{\frac{e^{\frac{1}{2 n}}-1}{\frac{1}{2 n}}+1}$. Therefore, $p^{\prime}\left(y_{0}\right)=0$ for $y_{0} \in(0,1)$ such that

$$
y_{0}^{\frac{e^{\frac{1}{2 n}}-1}{\frac{1}{2 n}}+1}=\frac{1+e^{\frac{1}{2 n}}}{2 e^{\frac{1}{2 n}}}
$$

Now, (13) and (14) imply

$$
\begin{aligned}
p_{\max }\left(y_{0}\right) & =y_{0}\left(1-y_{0}^{\frac{e^{\frac{1}{2 n}}-1}{2 n}}+1\right. \\
& =y_{0}\left(1-\frac{1+e^{\frac{1}{2 n}}}{2 e^{\frac{1}{2 n}}}\right)=y_{0} \frac{e^{\frac{1}{2 n}}-1}{2 e^{\frac{1}{2 n}}} .
\end{aligned}
$$

It is easy to verify that for $0<z \leq \frac{1}{2}$

$$
e^{z}-1 \leq 2 z \text {. }
$$

Consequently, for $z=\frac{1}{2 n}$,

$$
e^{\frac{1}{2 n}}-1<\frac{1}{n}
$$

Hence, (15) implies

$$
p_{\max }\left(y_{0}\right) \leq y_{0} \frac{1}{2 n e^{\frac{1}{2 n}}}<y_{0} \frac{1}{2 n}<\frac{1}{2 n} .
$$

Therefore, (11), (13), (15), (17) yield (9).

\section{Direct Quantitative Estimate for $M_{n}$}

Our goal in this section is to establish a full adequate and compatible quantitative estimate for approximation by the operator $M_{n}$, applied to functions $f \in C_{\varphi}^{k}\left(\mathbb{R}^{+}\right)$.

Such quantitative direct results should have the following form:

For all $f \in C_{\varphi}^{k}\left(\mathbb{R}^{+}\right), x \geq 0, \delta>0$ the following estimate holds true

$$
\left\|M_{n} f-f\right\|_{\varphi} \leq A_{f} \omega_{\varphi}(f, \delta),
$$

where $\delta=\delta_{n} \rightarrow 0$ when $n \rightarrow \infty$, and $A_{f}$ is a positive constant, independent of $n, x$, eventually depending only on $f$.

Unfortunately, we are not able to establish (18). Instead of this, we prove the following quantitative direct estimate.

Theorem 3. For all $f \in C_{\varphi}^{k}\left(\mathbb{R}^{+}\right), x \geq 0, n \in \mathbb{N}$, the following estimate holds true

$$
\left\|M_{n} f-f\right\|_{\varphi^{5 / 2}} \leq 6 \omega_{\varphi}\left(f, \frac{1}{\sqrt{n}}\right) .
$$

Proof. From ([28], Lemma 3, p. 104), we have for every $f \in C_{\varphi}\left(\mathbb{R}^{+}\right)$, for $\delta>0$ and for all $x, t \geq 0$

$$
|f(t)-f(x)| \leq(\varphi(t)+\varphi(x))\left[2+\frac{\left|e^{\frac{t}{2}}-e^{\frac{x}{2}}\right|}{\delta}\right] \omega_{\varphi}(f, \delta) .
$$


We apply $M_{n}$ to both sides of (20), and using (6), we obtain

$$
\left|M_{n}(f ; x)-f(x)\right| \leq\left[4 \varphi(x)+\varphi(x) \frac{M_{n}\left(\left|e^{\frac{t}{2}}-e^{\frac{x}{2}}\right| ; x\right)}{\delta}+\frac{M_{n}\left(\varphi(t)\left|e^{\frac{t}{2}}-e^{\frac{x}{2}}\right| ; x\right)}{\delta}\right] \omega_{\varphi}(f, \delta) .
$$

Using Cauchy-Schwarz inequality, we obtain

$$
\begin{aligned}
M_{n}\left(\left|e^{\frac{t}{2}}-e^{\frac{x}{2}}\right| ; x\right) & \leq \sqrt{M_{n}\left(\left(e^{t}+e^{x}-2 e^{\frac{x}{2}} \cdot e^{\frac{t}{2}}\right) ; x\right)} \\
& =\sqrt{M_{n}\left(2 e^{\frac{x}{2}}\left(e^{\frac{x}{2}}-e^{\frac{t}{2}}\right) ; x\right)} .
\end{aligned}
$$

From (11) and (15), we obtain

$$
M_{n}\left(e^{\frac{t}{2}}-e^{\frac{x}{2}} ; x\right) \leq\left(1+e^{x}\right) \frac{e^{\frac{1}{2 n}}-1}{2 e^{\frac{1}{2 n}}}<\left(1+e^{x}\right) \frac{1}{2 n e^{\frac{1}{2 n}}}<\frac{1}{2 n} .
$$

Therefore,

$$
M_{n}\left(\left|e^{\frac{t}{2}}-e^{\frac{x}{2}}\right| ; x\right) \leq \sqrt{e^{\frac{x}{2}}\left(1+e^{x}\right) \cdot \frac{1}{n}} \leq \frac{1}{\sqrt{n}} \frac{\varphi(x)}{\sqrt{2}} .
$$

In a similar way, we proceed with the second ratio in (21) again using Cauchy-Schwarz inequality

$$
\begin{aligned}
M_{n}\left(\varphi(t)\left|e^{\frac{t}{2}}-e^{\frac{x}{2}}\right| ; x\right) & \leq \sqrt{M_{n}\left(\varphi^{2}(t) ; x\right)} \cdot \sqrt{M_{n}\left(\left|e^{\frac{t}{2}}-e^{\frac{x}{2}}\right|^{2} ; x\right)} \\
& \leq \sqrt{M_{n}\left(\left(1+e^{t}+e^{2 t}\right) ; x\right)} \frac{\varphi(x)}{\sqrt{2 n}}
\end{aligned}
$$

Using (5), we calculate

$$
M_{n}\left(e^{2 t} ; x\right)=e^{x} R_{n, 2}^{*}\left(e^{t}, x\right)=e^{x\left(1+e^{\frac{1}{n}}\right)} .
$$

Therefore,

$$
\left.M_{n}\left(1+e^{t}+e^{2 t} ; x\right)=1+e^{x}+e^{x\left(1+e^{\frac{1}{n}}\right.}\right) \leq\left(1+e^{x}\right)^{3}, n \geq 2 .
$$

Now, (23) and (24) imply

$$
M_{n}\left(\varphi(t)\left|e^{\frac{t}{2}}-e^{\frac{x}{2}}\right| ; x\right) \leq \frac{\varphi^{5 / 2}(x)}{\sqrt{2 n}} .
$$

Hence, (21)-(23) and (25) lead to

$$
\left|M_{n}(f ; x)-f(x)\right| \leq\left[4+\frac{1}{\delta \sqrt{2 n}}+\frac{1}{\delta \sqrt{n}} \sqrt{\frac{5}{2}}\right] \omega_{\varphi}(f, \delta) .
$$

We set in (26) $\delta=\frac{1}{\sqrt{n}}$ and obtain

$$
\left|M_{n}(f ; x)-f(x)\right| \leq 6 \varphi^{5 / 2}(x) \omega_{\varphi}\left(f, \frac{1}{\sqrt{n}}\right) .
$$


Since $\|f\|_{\varphi^{5 / 2}}=\sup _{x \geq 0} \frac{|f(x)|}{\varphi^{5 / 2}(x)}$, it is clear that

$$
\left\|M_{n} f-f\right\|_{\varphi^{5 / 2}} \leq 6 \omega_{\varphi}\left(f, \frac{1}{\sqrt{n}}\right) .
$$

The proof of Theorem 3 is completed.

Remark 1. The proofs of Theorems 2 and 3 rely on some ideas from [26,28]. For our operator $M_{n}$ introduced to approximate functions $f \in C_{\varphi}\left(\mathbb{R}^{+}\right)$with exponential growth, we can not apply Theorem 3 and Corollary 1 in [28] due to the fact that $d_{n}=\left\|M_{n} \varphi^{3}-\varphi^{3}\right\|_{\rho^{\frac{3}{2}}} \rightarrow \infty$, when $x \rightarrow \infty$ (with notation from [28], $\rho(x)=1+\varphi^{2}(x)$ ).

Remark 2. In [11], Aral, Inoan and Raşa studied approximation properties of some generalized Szász-Mirakyan operators and obtained in ([11], Theorem 2.2) the following quantitative estimate

$$
\left\|S_{n}^{\rho}(f)-f\right\|_{\varphi^{3 / 2}} \leq\left(7+\frac{2}{n}\right) \omega_{\varphi}\left(f ; \frac{2}{\sqrt{n}}+\frac{7}{n}\right),
$$

where $\varphi(x)=1+\rho^{2}(x)$ and $\rho(x)=x+x^{2}, x \in(0, \infty)$, that is, they considered approximation of unbounded functions with polynomial growth. In this sense, our paper is an extension of [11] for functions with exponential growth.

Remark 3. Our direct quantitative estimate in Theorem 3 improves the result of Theorem 2 in [2], where another modification of Szász-Mirakyan-type operators was considered, which fix $e^{a x}$ and $e^{2 a x}$ with $a>0$.

Conjecture 1. With notations given in Section 1, the quantitative estimate (18) holds true either for the operator $M_{n}$ or for some other variants of Szász-Mirakyan, Baskakov, Phillips, etc., operators, which fix certain exponential functions.

\section{Conclusions and Perspectives}

In the last years, many papers concerning positive linear operators which preserve exponential functions were published. Combining two techniques, a new procedure to construct sequences of positive linear operators fixing exponential functions is described. Our results improve and extend similar results on this topic, and we mentioned here the papers [2,11,26]. The studies on Szász-Mirakyan operators considered in [26] are continued, with results concerning uniform weighted convergence. At the end of the paper, we propose to the reader a conjecture concerning the rate of approximation by certain operators which fix some exponential functions.

Author Contributions: These authors contributed equally to this work. All authors have read and agreed to the published version of the manuscript.

Funding: Project financed by Lucian Blaga University of Sibiu \& Hasso Plattner Foundation research grants LBUS-IRG-2021-07.

Conflicts of Interest: The authors declare no conflict of interest.

\section{References}

1. King, J.P. Positive linear operators which preserve $x^{2}$. Acta Math. Hung. 2003, 99, 203-208. [CrossRef]

2. Acar, T.; Aral, A.; Cárdenas-Morales, D.; Garrancho, P. Szász-Mirakyan type operators which fix exponentials. Results Math. 2017, 72, 1393-1404. [CrossRef]

3. Acar, T. Asymptotic formulas for generalized Szász-Mirakyan operators. Appl. Math. Lett. 2015, 263, 223-239. [CrossRef]

4. Duman, O.; Özarslan, M.A.; Della Vechia, B. Modified Szász-Mirakyan-Kantorovich operators preserving linear functions. Turk. J. Math. 2009, 33, 151-158. 
5. Yilmaz, O.G.; Bodur, M.; Aral, A. On approximation properties of Baskakov-Schurer-Szász operators preserving exponential functions. Filomat 2018, 32, 5433-5440. [CrossRef]

6. Aral, A.; Cardenas-Morales, D.; Garrancho, P. Bernstein type operators that reproduce exponential functions. J. Math. Inequal. 2018, 12, 861-872. [CrossRef]

7. Aral, A.; Ulusoy, G.; Deniz, E. A new construction of Szász-Mirakyan operators. Num. Algorithms 2018, 77, 313-326. [CrossRef]

8. Deniz, E.; Aral, A.; Gupta, V. Note on Szász-Mirakyan-Durrmeyer operators preserving $e^{2 a x}, a>0$. Num. Funct. Anal. Optim. 2018, 39, 201-207. [CrossRef]

9. Bodur, M.; Yilmar, O.G.; Aral, A. Approximation by Baskakov-Szász-Stancu operators preserving exponential functions. Constr. Math. Anal. 2018, 1, 1-8. [CrossRef]

10. Cárdenas-Morales, D.; Garrancho, P.; Raşa, I. Bernstein-type operators which preserve polynomials. Compt. Math. Appl. 2011, 62, 158-163. [CrossRef]

11. Aral, A.; Inoan, D.; Raşa, I. On the generalized Szász-Mirakyan operators. Results Math. 2014, 65, 441-452. [CrossRef]

12. Duman, O.; Özarslan, M.A. Szász-Myrakyan type operators providing a better error estimation. Appl. Math. Lett. 2007, 20, 1184-1188. [CrossRef]

13. Gupta, V. Approximation by Generalizations of Hybrid Baskakov Type Operators Preserving Exponential Functions, Mathematical Analysis and Applications: Selected Topics; Michael, R., Hemen, D., Ravi, P.A., Eds.; John Wiley \& Sons, Inc.: Hoboken, NJ, USA, 2018.

14. Gupta, V.; Acu, A.M. On Baskakov-Szász-Mirakyan-type operators preserving exponential type functions. Positivity 2018, 22, 919-929. [CrossRef]

15. Gupta, V.; Aral, A. A note on Szaász-Mirakyan-Kantorovich type operators preserving $e^{-x}$. Positivity 2018, 22, 415-423. [CrossRef]

16. Gupta, V.; Tachev, G. On approximation properties of Phillips operators preserving exponential functions. Mediterr. J. Math. 2017, 14, 177. [CrossRef]

17. Ulusoy, G.; Acar, T. Approximation by modified Szász-Durrmeyer operators. Period. Math. Hung. 2016, 72, 64-75.

18. Boyanov, B.D.; Veselinov, V.M. A note on the approximation of functions in an infinite interval by linear positive operators. Bull. Math. Soc. Sci. Math. Roum. 1970, 14, 9-13.

19. Holhoş, A. The rate of approximation of functions in an infinite interval by positive linear operators. Stud. Univ. Babeş-Bolyai Math. 2010, 2, 133-142.

20. Yilmaz, Ö.G.; Gupta, V.; Aral, A. On Baskakov operators preserving the exponential functions. J. Numer. Anal. Approx. Theory 2017, 46, 150-161.

21. Yilmaz, Ö.G.; Aral, A.; Yesildal, F.T. On Szász-Mirakyan type operators preserving polynomials. J. Numer. Anal. Approx. Theory 2017, 46, 93-106.

22. Yilmaz, Ö.G.; Gupta, V.; Aral, A. A note on Baskakov-Kantorovich type operators preserving $e^{-x}$. Math. Methods Appl. Sci. 2020, 43, 7511-7517. [CrossRef]

23. Gupta, V.; López-Moreno, A.J. Phillips operators preserving arbitrary exponential functions $e^{a t}, e^{b t}$. Filomat 2018, 32, 5071-5082. [CrossRef]

24. Gadziev, A.D. The convergence problem for a sequence of positive linear operators on unbounded sets and theorems analogues to that of P.P. Korovkin. Dokl. Akad. Nauk. SSSR 1974, 218, 1001-1004.

25. Mahmudov, N.I. q-Szász-Mirakyan operators which fix $x^{2}$. J. Comput. Appl. Math. 2011, 235, 4621-4628. [CrossRef]

26. Acar, T.; Aral, A.; Gonska, H. On Szász-Mirakyan operator preserving $e^{2 a x}, a>0$. Mediterr. J. Math. 2017, 14, 6. [CrossRef]

27. Gadziev, A.D. Theorems of the type of P.P. Korovkin's Theorems. Mat. Zametki 1976, 20, 781-786.

28. Holhoş, A. Quantitative estimates for positive linear operators in weighted spaces. Gen. Math. 2008, 16, 99-110. 